Review

International Journal of Biological Sciences

ISSN 1449-2288 www.biolsci.org 2007 3(3):185-191

(c) Ivyspring International Publisher. All rights reserved

\title{
Identiifying the Future Needs for Long-Term USDA Eiforts in Agricultural Animal Genomics
}

\author{
R. D. Green ${ }^{1}$, M. A. Qureshi², J. A. Long ${ }^{3}$, P.J. Burfening², and D.L. Hamernik² \\ 1. USDA-ARS, National Program Staff, Beltsville, Maryland, USA 20705 \\ 2. USDA-CSREES, National Program Staff, Washington, District of Columbia, USA 20250 \\ 3. USDA-ARS Biotechnology and Germplasm Laboratory, Beltsville, Maryland, USA 20705
}

Correspondence to: Debora L. Hamernik; United States Department of Agriculture; Cooperative State Research, Education, and Extension Service; 1400 Independence Avenue, SW; Stop 2220; Washington, DC 20250-2220; USA E-mail: dhamernik@csrees.usda.gov; Tel: (202)401-4202; Fax: (202)401-1602

Received: 2006.12.14; Accepted: 2007.01.25; Published: 2007.02.10

Agricultural animal research has been immensely successful over the past century in developing technology and methodologies that have dramatically enhanced production efficiency of the beef, dairy, swine, poultry, sheep, and aquaculture industries. In the past two decades, molecular biology has changed the face of agricultural animal research, primarily in the arena of genomics and the relatively new offshoot areas of functional genomics, proteomics, transcriptomics, metabolomics and metagenomics. Publication of genetic and physical genome maps in the past 15 years has given rise to the possibility of being able finally to understand the molecular nature of the genetic component of phenotypic variation. While quantitative geneticists have been remarkably successful in improving production traits, genomic technology holds potential for being able to lead to more accurate and rapid animal improvement, especially for phenotypic traits that are difficult to measure.

Recently, the agricultural research community has been able to capitalize on the infrastructure built by the human genome project by sequencing two of the major livestock genomes (Gallus domesticus and Bos Taurus). The 2005 calendar year is truly unprecedented in the history of agricultural animal research since draft genome sequences were completed for chickens and cattle. In addition, sequencing the swine and equine genome was initiated in early 2006. We now have in place a powerful toolbox for understanding the genetic variation underlying economically important and complex phenotypes.

Over the past few years, new challenges have emerged for animal agriculture. Enhancements in production efficiency have not come without some negative side effects on animal well-being and longevity in production environments, including losses in reproductive efficiency, increased stress susceptibility, increased animal waste issues, and increased susceptibility to animal metabolic and infectious diseases. When considered in concert with societal concerns in the areas of natural resource conservation and protection, animal welfare, and food safety, it is clear that publicly supported agricultural research must be focused on enhancing the functionality and well-being of livestock and poultry in environmentally neutral production systems in the future.

Realizing the great potential for animal genomics to address these and other issues, a workshop was convened by the U. S. Department of Agriculture (USDA) in Washington, DC in September of 2004. The workshop was entitled "Charting the Road Map for Long Term USDA Efforts in Agricultural Animal Genomics". This paper summarizes the proceedings of the workshop and the resulting recommendations. The need for a cohesive, comprehensive long-term plan for all of USDA's research efforts in animal genomics was evident at the workshop, requiring further integration of the efforts of the USDA's Cooperative State Research, Education, and Extension Service (CSREES) and the USDA's Agricultural Research Service (ARS) to achieve the greatest return on investment.

Key words: Animal Genomics; Sequencing, Functional Genomics, Bioinformatics

\section{Introduction}

In 2001, representatives from several universities, private industries, producer groups, and scientific societies formed the Alliance for Animal Genome Research (the Alliance) to advocate for public funding for domestic animal genomics research. The Alliance worked with the National Academy of Sciences to organize a public workshop in 2002 entitled "Exploring Horizons for Domestic Animal Genomics" [6]. The goal of the workshop was to identify research goals and pub- lic and private funding to advance domestic animal genomics. This workshop drew upon experts from both human and agricultural genomics and built upon recommendations from earlier planning workshops. There was overwhelming consensus at the workshop that funding should be identified to produce high-coverage, draft genome sequences of the major domestic animal species (cattle, chicken, swine, dog, and cat). The National Institutes of Health's (NIH) National Human Genome Research Institute (NHGRI) 
had previously established a process for prioritizing species for sequencing based upon the ability of a species to better inform annotation of the human genome sequence through evolutionary comparisons. The workshop participants felt that cattle, chicken, swine, dog, and cat would be excellent candidates for sequencing because these species had been used heavily as biomedical models and they were important food or companion animal species. Some of the workshop participants also expressed enthusiasm for sequencing fish or the honeybee because these agriculturally important species are also powerful tools for fine mapping of quantitative trait loci (QTL). Furthermore, it was recommended that there would need to be appropriate scaling-up of bioinformatics resources and public databases to make effective use of the information that would result from the genome sequencing projects. Based upon the experiences of the National Plant Genome Initiative, it was also recommended that funding for such large-scale projects would need to come from a variety of sources, including the U.S. Federal government, private industry, and international partners.

In response to a request by the then USDA Undersecretary Joseph Jen, a new Interagency Working Group (IWG) on Domestic Animal Genomics was chartered in September of 2002 by the U.S. National Science and Technology Council. The membership of the IWG includes representatives from the USDA, Department of Energy (DOE), Food and Drug Administration (FDA), NIH, National Science Foundation (NSF), Office of Science and Technology Policy (OSTP), Office of Management and Budget (OMB), Department of Homeland Security (DHS) and the U.S. Agency for International Development (USAID). The IWG determined that large-scale sequencing, data management and bioinformatics, and functional genomics were the specific goals to be achieved in fiscal years 2003 to 2007. The IWG recommended:

- Large-scale sequencing to produce draft genome sequences (8-fold sequence coverage) of honeybee, chicken, canine, bovine, swine, and feline species.

- Data management and bioinformatics to specifically support agriculturally important species, including significant improvements in data management and analysis software, allow for greater data accessibility and secure long term maintenance, increase capabilities to deal with rapidly accumulating data complexity as databases include functional information, and provide more powerful tools to mine large genomes.

- Recognition that an increase in data for livestock genomes requires a concomitant investment in functional genomics to support genome annotation, the study of gene regulation and expression, and species evolutionary relationships.

Since 2002, considerable progress has been achieved toward the goal of placing whole genome sequence and associated genomic tools into the public domain for high priority domestic animal species. Annotated draft sequences have been published for the honeybee, chicken, and dog genomes. The bovine genome sequencing project has reached $>6$-fold coverage and is entering the final gene prediction and annotation phase. Lighter coverage sequencing of the cat genome has been completed. Sequencing of the swine genome was initiated in 2006. Developed concomitantly with these genome projects has been a suite of associated tools including EST libraries, BAC maps, integrated physical and linkage maps, full-length cDNA libraries, microarrays or gene chips, and identification and validation of a large number of single nucleotide polymorphism (SNP) markers. All of these efforts have required leveraging of efforts between agriculture and the biomedical sciences, as well as unprecedented partnerships between U.S. Federal research agencies, international groups, universities, and private industry.

In early 2004 as the sequencing goals of the IWG appeared to be within reach, further strategic planning for how to best address the remaining two areas of greatest importance - bioinformatics and functional genomics - was warranted. Specifically, the IWG charged the USDA to identify future needs for long-term efforts in agricultural animal genomics to allow full utilization of annotated genome sequences and associated genomic tools.

\section{USDA animal genomics workshop overview}

Life sciences research activities in the USDA are administered by two separate agencies. The CSREES funds extramural research efforts conducted primarily at land grant universities. The ARS is the intramural research arm of the USDA and funds long-term, high-risk research on an ongoing basis in its 108 labs throughout the U.S. In fiscal year 2005, USDA funding for animal genomics research totaled \$51.3M (ARS $\$ 23.5 \mathrm{M}$ and CSREES - \$27.8M).

The USDA Animal Genomics Workshop provided an opportunity for USDA administrators to interact with leading scientists in the field of food animal genomics to identify research needs and priorities in the post-genome sequencing era. Participating academic or ARS scientists were selected to reflect a balance of funding sources (16 from CSREES and 18 from ARS), species of primary interest (balance between poultry, swine, cattle, sheep, and aquaculture), and area of research emphasis or expertise (gene mapping, bioinformatics and statistical genetics, functional genomics). In addition to a number of program administrators from ARS and CSREES, colleagues from NIH, NSF, and DHS also participated in the workshop.

\section{Priorities for Structural Genomics in Domestic Animal Genomics.}

The opening session focused on the needs for structural genomics faced by animal genomics researchers today. Generally, scientists have approached genomics by building structural genomics resources, with ventures into functional genomics observed only in more recent years. Through international collaborations and efforts, linkage and comparative maps for all livestock species have been made available to the pub- 
lic. The recent and ongoing development of whole genome sequence maps of the chicken, honeybee, dog, and cattle species is a major step forward. SNP based-maps now being developed from the chicken and cattle genome sequencing projects will be of enormous value in evaluating genetic diversity, fine mapping of QTL, and development of DNA-based animal identification systems. While the current trend toward internalization of genomics research in private companies indicates the potential value of genomic tools, it also was pointed out as a major concern. There was a consensus that we must complete the basic genome infrastructure for all major species and deposit such information in public databases to facilitate rapid discovery and the development of commercially usable technologies for agricultural and biomedical sciences and industries.

A major advantage of using agricultural animal species in genomics research is the widespread availability of large, pedigreed animal populations for research. Many of these populations have been in existence for fifty years or more and have been phenotyped widely for a variety of economically and biologically important characteristics. In the past two decades, a number of sub-populations were set up as resource families for use in QTL detection and subsequently for validation of putative QTL. Participants agreed that it is imperative in the post-genome sequencing era that the value of these populations, and tissue repositories derived from them, be recognized and supported.

Participants agreed that animal genomics is poised to impact several avenues of animal production, life sciences, and biomedical research; however, physical and financial resources are crucial to capitalizing on past investments. The utilization of resources and human capital must be carefully directed toward achieving outcomes and deliverables that are measurable in application, promote rapid commercialization, and enhance education of the public and the next generation of scientists.

Specific Recommendations from the Structural Genomics Module:

1. Sequence the swine genome to a minimum of 6-fold coverage for deposit into the public domain.

2. Obtain BAC maps and 2-fold sequence coverage, develop comprehensive full-length cDNA libraries to allow functional annotation, and complete integration of genetic linkage, radiation hybrid and physical maps for the catfish, goat, horse, salmon, trout, and turkey genomes.

3. Discover and validate SNP markers and develop haplotype maps for all species to increase the density of maps for fine mapping of QTL and eventual "whole genome selection".

4. Develop standardized population and phenotype resources for each of the species. a). Preserve long-term, unique, experimental animal populations to capitalize on their value in functional genomics research and further develop and maintain diverse animal resource families. b). Couple these animal popu- lations with genotypic and phenotypic information and obtain funding support for appropriate long-term tissue repositories for tissue cultures, DNA and RNA. c). Explore options to develop a centralized facility with sufficient technical expertise to preserve unique experimental food animal populations, collect genotypic and phenotypic information on these animals, and make the populations available to the agricultural and biomedical research communities (i.e. the Jackson Labs model).

\section{Priorities for Functional Genomics in Domestic Animal Genomics.}

The second module of the workshop was an open discussion of the challenges facing agricultural animal genomics researchers in capitalizing on the structural genomics infrastructure through downstream applications in functional genomics, proteomics, metabolomics, and metagenomics.

The "grand challenges" are: 1) functional annotation of genes; 2) complete description and understanding of cellular pathways (e.g., metabolism, proliferation, differentiation, cell-cell interaction); 3) genomic-environment interaction (e.g., developmental pathways, abiotic stresses such as heat, cold, and drought, nutritional genomics, and infectious diseases); 4) the development of an encyclopedia of economic trait loci, and 4) understanding and applying the heritable variation in the genome. A need for additional biological resources (e.g., tissue banks, animal germplasm, cell lines), genomic technologies (e.g., RNAi, genotyping services, cloning and transgenics) and integrative databases and informatics was identified.

General Discussion. Downstream or post-sequence activities, such as functional genomics, proteomics and metabolomics, clearly are the areas where agricultural species will benefit from the initial investments in genome sequencing. For both human and agricultural species, the post-sequencing challenge will be to understand the operation and function of genomic information. In particular, the primary issue for agricultural species will be translating the respective genome sequences into enhanced value of the phenotypes (e.g., disease resistance, behavior, growth, product quality, reproduction).

The post-sequencing era will move rapidly from crudely defined genomic relationships with phenotypes, such as QTL, to a rapid dissection of those relationships in the context of true functional genomics. Some examples of QTL that should progress rapidly from chromosomal localization to industrial application include meat quality and product yield in beef cattle, milk production and mastitis resistance in dairy cattle, litter size and uterine capacity in swine, product yield and parasite resistance in sheep, and coccidia resistance in poultry. The availability of genome sequences for agricultural species is significantly enhancing fine mapping of individual genes in two key ways. First, an exponential increase in the numbers of SNPs distributed throughout the linkage maps are 
enabling fine mapping of QTL at a level that was not possible previously. For example, poultry genomics is poised to realize this benefit with the placement of some 3 million SNPs on a 1.2 Gb genome. Second, comparative genomics will increase the likelihood of QTL identification by virtue of the highly conserved regions of genes throughout mammalian species (e.g., myostatin gene responsible for double-muscling condition in cattle $[7,8,9])$.

The majority of economically important traits exhibit complex or multifactorial inheritance patterns that are influenced by environmental factors; therefore, the principal challenge is not simply detecting the QTL, but rather unraveling the genes and the regulatory elements that control gene expression [10]. This will require the integration of numerous resources, including genetic and physical maps, QTL markers, EST libraries, microarrays and the whole genome sequence to delineate the molecular mechanisms that control complex biological systems. Agricultural species have an advantage in that phenotypes are well characterized and diverse because they have been closely monitored and specifically modified through selected breeding, both in private industry and in experimental populations.

Expression profiling of large numbers of genes across diverse tissues, populations, and environmental states also will use increasingly sophisticated quantification platforms. For example, the expression of literally thousands of genes can be studied simultaneously already using DNA chips or microarrays. The molecular biologist will be able to bypass traditional laborious processes, such as screening BAC libraries, and instead clone genes "in silico" [11]. Proteomic technologies, including new developments in mass spectrometry and database searching, are leading to rapid advances in monitoring genome activity at the protein level. We can expect further advances in understanding the structural biology of proteins when comparative and evolutionary approaches to sequencing are utilized. Proteome analysis will elucidate groupings of genes that regulate metabolic pathways. Additionally, by following gene expression fluctuations over time and in response to specific signals, the position occupied by the protein end product of a particular gene, relative to others in metabolic and signaling pathways, can be inferred [12]. It follows, then, that fields, such as metabolomics, will allow genomic characterization of "systems" of proteins and their applications to animal health and nutrition, as well as human nutrition and obesity. Whereas genes and proteins set the stage for what happens in the cell, much of the actual activity is at the metabolite level: cell signaling, energy transfer, and cell-to-cell communication are all regulated by metabolites [13].

New technologies will continue to be developed at a rapid pace to improve both the precision and efficiency of the various 'omics' approaches. For instance, the phenomenon of RNA interference (RNAi) has evolved rapidly into a powerful technique to silence gene expression in eukaryotic cells. Because RNAi technology can be used to knock out genes across a genome, having the complete genome sequence will greatly improve identification of 'targets' (proteins) for existing drugs. Another emerging technology, metagenomics, is poised to develop rapidly and have profound impacts on functional genomics research in agricultural species. Metagenomics is a new field combining molecular biology and genetics in an attempt to identify and characterize the genetic material from environmental samples and apply that knowledge. Genetic diversity is assessed by isolation of DNA followed by direct cloning of functional genes from the environmental sample. The metagenomics field was pioneered when researchers used whole genome shotgun sequencing to sequence microbial populations en masse from the Sargasso Sea [14]. It is not hard to envision application of this technology to ascertain the microbial populations of the bovine rumen or monogastric intestine, for example, and how the dynamics/interactions among bacterial and protozoan species create a unique microenvironment that promotes growth.

Perhaps the most intriguing example of new technology development on the heels of genome sequencing has been the NHGRI's investment in the next generation of DNA sequencing technologies. The goal of this NHGRI effort is to lower the cost of sequencing an individual's genome (human or animal) to $\$ 1000$ (USD). Once in place, these technologies will further revolutionize the post-sequencing era for agricultural animal species to allow genome sequencing of additional minor species as well as sequencing of individual animal genomes.

With all of the expected and rapid increases in knowledge in the near future, it is imperative that the methodology for defining phenotypes be clear and standardized. More importantly, any search for mutations or altered functional expression depends on phenotypic screening and the ability to detect variation from normal. The challenge, then, is to develop efficient, systematic, and comprehensive phenotypic screening procedures and tools that will permit comparison among laboratories. For example, the current phenotypes of highly pathogenic avian influenza (HPAI) were formulated over 10 years ago when the only virus known to have mutated to virulence was the HPAI responsible for the 1983-84 Pennsylvania epizootic [15]. Cumulative evidence, however, suggests that HPAI viruses actually arose from low-pathogenicity avian influenza (LPAI) H5 or H7 viruses infecting poultry after spreading from free-living birds. At present, it can only be assumed that all $\mathrm{H} 5$ and $\mathrm{H} 7$ viruses have this potential and mutation to virulence is a random event. Therefore, the longer the presence and greater the spread in poultry, the more likely it is that HPAI virus will emerge [15]. This example illustrates how major research efforts in phenotypic screening are needed to characterize traits that have been difficult to measure until now.

Concomitant with the advent of functional ge- 
nomics, the types and amounts of data that need to be stored in databases have changed dramatically. Many types of information that were previously collected on an ad hoc basis now need to be stored in a more structured manner. Additional data sets for gene expression, proteomics, and protein-protein interactions are growing increasingly complex. To analyze data computationally in an efficient manner, there is a need for consistency between expressions in different phenotypic domains as well as in different species. The term "phenotype" can be used in different ways in different fields in biology and by different researchers in those fields. The development of phenotypic ontologies for livestock is critical to connect heterogeneous data types back to the animal.

Equally important is to approach functional genomics, proteomics and metabolomics from an integrative systems biology perspective. Within a systems biology approach, each type of biological information (DNA, RNA, protein, protein interactions, biomodules, cells, tissues, etc.) also has individual elements (e.g., specific promoters, genes or proteins), and the interrelationships of all these elements and types of biological information must be determined and integrated to obtain a view of the system as a whole. What is ultimately desired is the ability to unravel the complexities of epistatic and genotype by environment interactions and how they affect phenotypic expression. Deciphering these complexities requires a holistic approach that describes and understands the biology underlying phenotypes.

The post-genome sequencing era will bring enormous quantitative and phenotypic data to the table. The USDA is the logical organization to lead this systems biology approach for agricultural species. It was suggested that compartmentalization of genomics programs, as has been done in the past for both CSREES and ARS program management, should be shifted toward integration of functional genomics approaches into all program areas and disciplines (e.g., animal growth and production, animal health, animal well-being, aquaculture, food safety, animal waste management, animal and human nutrition, etc.). A cross-disciplinary research effort will be required to integrate the global genomics data into information that is usable and applicable across the diverse landscape of agricultural production.

Specific Conclusions and Recommendations from the Functional Genomics Module:

1. Downstream work in functional genomics and proteomics will be where the benefits from animal genomics research are reaped.

2. Develop a clear and standardized methodology for defining phenotypes, particularly in the emerging areas of animal health and well-being.

3. Agricultural animal genomics research is ideally suited to the integrative systems biology "big science" approach required to unravel the complexity of epistatic and genotype by environment interactions.

4. Significantly enhance the bioinformatics capacity within the public agricultural animal research enterprise to handle the increasing complexity and volume of genomic and proteomic data.

5. Make downstream functional genomics, proteomics, metabolomics, and metagenomics research in agricultural animal species a high priority to capitalize on the previous investments in genomic resources, tools, and reagents.

6 . End the previous separation of genomics efforts within USDA research portfolios and tegrate functional genomics approaches as a foundation in all program areas and disciplines.

7. To integrate genomic approaches across disciplines, improve the coordination and effectiveness between ARS and CSREES by developing and implementing a long-term strategic plan for USDA animal genomics research.

\section{Priorities for Bioinformatics Resources in Domestic Animal Genomics.}

The third and final module of the workshop focused on bioinformatics needs. The most useful current databases and bioinformatics resources for agricultural animal genomics are those at The Institute for Genomics Research (TIGR), National Center for Biotechnology Information (NCBI), MS-Access or MySQL software for local sequence and genotyping databases, and SeqWeb. In addition, the Generic Model Organism Database (GMOD), funded by the NIH and the ARS, was mentioned as a unique tool for genome database visualization, curation, and ontology. Adequate databases and tools are available to manage and analyze ESTs, SNPs, microarray, SAGE and proteomics information; however, there remain unique personnel, skills, and software needs for each of these tools. It was noted that the general lack of bioinformatics personnel and minimal integration of relational databases with all aspects of research are the two critical factors that are limiting progress in the field of bioinformatics.

Specific Recommendations from the Bioinformatics Module:

1. Focus USDA resources on its unique capabilities, such as phenotypic characterization, population and quantitative genetics, physiology, etc., and be careful to not "re-invent" the bioinformatics capabilities already in place in other genomics research communities.

2. Immediately provide training programs and associated support for faculty sabbaticals, postdoctoral associates, and graduate students focused on integrating biology and computing, since one rate-limiting step for USDA in bioinformatics is awareness and literacy in use of existing tools and lack of basic training programs to bring new bioinformatics personnel online.

3. Develop standard descriptions of phenotypes as this is a second rate-limiting step for USDA in bioinformatics and it is a problem that will be exacerbated when functional genomics research moves into the more challenging areas of animal health and well-being in the near future. 
4. Create a USDA bioinformatics working group at the Research, Education, and Economics mission area level to: a) coordinate and define ARS and CSREES efforts among data producers, tool developers, analysts, and consumers; and b) better coordinate with other Federal and international agencies.

5 . To best serve the bioinformatics needs of agricultural animal genomics, leverage USDA resources with others to develop expertise and new tools. a. Support species-specific annotation; b. Organize curation groups for management of livestock genome sequence resources, in concert with existing groups (i.e,. NCBI, UCSC, Ensembl), to help build browsers with characteristics important to current and future animal genomics research. c. Link animal genomic data to published literature in the animal sciences. d. As large numbers of SNP are discovered and validated, develop databases linking haplotypes with phenotypes and further tools (e.g., NCBI, dbSNP) to facilitate QTL mapping and association studies for multiple species. e. Develop a centralized and standardized system for microarray analysis and gene expression databases by requiring agreement on database platform (s) for microarray target annotation and gene expression data mining with intentions to link to genome assemblies and associated gene and protein databases.

\section{Conclusions}

There is little doubt that the investments made to date in animal genomics will yield enormous dividends in the future for the producers and consumers of animal products and for the biomedical sciences. However, this workshop clearly identified a number of areas that need significant programmatic and administrative support within the USDA research infrastructure for this potential to be realized in a timely manner. Given the wealth of genotypic and phenotypic information catalogued on pedigreed agricultural animal populations, opportunities appear to exist and should be explored further for leveraging of future efforts with other Federal programs. Furthermore, there was strong consensus that in the post-sequencing era, research employing genomics techniques and tools should be integrated across all disciplines engaged in the animal sciences as opposed to being separated into "genomics" program areas. An overwhelmingly clear message from the workshop was that it is critical for USDA research leaders to develop and implement a visionary, long-term strategic plan for animal genomics research as soon as possible. Such a plan will ensure that the full potential of past, current, and future efforts and investments in animal genomics will have a positive impact on animal producers and the public in the post-sequencing era.

\section{USDA's implementation of workshop rec- ommendations}

In April of 2006, ARS and CSREES administrators formally launched the development of a long-term strategic plan in this area called the "USDA Blueprint for Animal Genomics". The "Blueprint" is ex- pected to be made available to the public on the ARS (www.ars.usda.gov) and CSREES (www.csrees.usda.gov) websites during the second quarter of 2007. Ancillary to this process was a Livestock Genomics Symposium held in July 2006 under the auspices of the US-European Commission's Task Force on Biotechnology where scientists and administrators from the US and a number of EC member countries identified priority areas where transatlantic cooperation could move this field forward [16].

Within ARS, the ARS National Program 101: Food Animal Production Action Plan 2007-2012 contains a significant emphasis on functional and structural genomics [17]. Additionally, components of the current 5-year action plans for ARS national programs in animal health (NP 103) and aquaculture (NP 106) also reflect the priorities identified in the USDA Workshop, particularly related to host:pathogen interactions that can be further elucidated via genomic approaches.

Within CSREES, the fiscal year 2006 Request for Applications (RFA) for the CSREES National Research Initiative (NRI) competitive grants program was the first opportunity to incorporate recommendations from this workshop into CSREES competitive programs. In fiscal year 2006, the RFA for the NRI Animal Genome program was subdivided into four sections: 1) Applied Animal Genomics, 2) Tools \& Resources, 3) Bioinformatics, and 4) Functional Animal Genomics. In addition, all applications submitted to one section of the NRI Animal Genome program were reviewed and ranked as a group by the peer review panel before reviewing applications submitted to one of the other sections of this program. Thus, in fiscal year 2006, the NRI Animal Genome program made the first-ever awards in animal bioinformatics (www.csrees.usda.gov/fo/animalgenomicsnri.html and click on "abstracts of funded projects"). In fiscal years 2006 and 2007, other disciplinary programs within the NRI (e.g., Animal Reproduction or Animal Growth and Nutrient Utilization) supported applications using gene transcription profiling (a component of functional genomics); these programs also required that applicants include physiological or functional studies at the cellular, systemic, or whole animal levels. In fiscal years 2006 and 2007, the NRI Animal Protection program also encouraged applicants to take advantage of genomic approaches (e.g., functional genomics, proteomics) in order to accelerate the discovery of new targets for diagnostics, vaccines, and treatments. Because it often takes applicants a couple of years to respond successfully to a new area in an RFA (i.e., applicants must collect sufficient preliminary data and write successful applications), it is anticipated that additional awards with functional genomics approaches will be made in other disciplinary programs within the NRI within the next five years.

\section{Acknowledgments}

The authors gratefully acknowledge the scientific contributions of D. Adelson, L. Alexander, S. Burgess, 
A. Capuco, H. Cheng, N. Cockett, L. Cogburn, E. Connor, P. Coussens, J. Dodgson, C. Elsik, C. Ernst, K. Eversole, L. Gasbarre, J. Keele, H. Lewin, H. Lillehoj, E. Marguiles, J. Ostell, J. Reecy, C. Rexroad, III, R.M. Roberts, G. Rohrer, M. Rothschild, D. Schneider, L. Schook, T. Smith, T. Sonstegard, J. Vallet, C. Van Tassell, G. Waldbeiser, W. Warren, J, Womack, K. Worley, and $\mathrm{K}$. Zuelke. Additional appreciation is extended to P. Brayton, L. Ellis, J. Jen, P. Johnsen, S. Kappes, M. Mirando, J. Peterson, and W. Zamer for their participation in the planning and implementation of the workshop. Finally, the efforts of the national program staff of CSREES, especially V. Martin, and ARS, especially L. Mangra, for handling the logistics of the workshop are sincerely appreciated.

\section{Conflict of interest}

The authors have declared that no conflict of interest exists.

\section{References}

1. Collins FS, Green ED, Guttmacher AE, Guyer MS. A vision for the future of genomics research. Nature 2003; 422:835-847.

2. International HapMap Consortium. A haplotype map of the human genome. Nature 2005; 437:1299-1320.

3. International Chicken Genome Consortium. Sequence and comparative analysis of the chicken genome provide unique perspectives on vertebrate evolution. Nature 2004; 432:695-716.

4. Wong GK, et al. International Chicken Polymorphism Consortium. A genetic variation map for chicken with 2.8 million single-nucleotide polymorphisms. Nature 2004; 432: 717-722.

5. [Internet] Gibbs et al. Bovine genomic sequencing initiative: De-humanizing the cattle genome. 2002. http://www.genome.gov/Pages/Research/Sequencing/SeqPr oposals/BovineSEQ.pdf

6. Pool R, Waddell K. Exploring Horizons for Domestic Animal Genomics: Workshop Summary. Washington DC, USA: National Academy Press; 2002.

7. Grobet L, Martin LJ, Poncelet D, Pirottin D, Brouwers B, Riquet J, Schoeberlein A, Dunner S, Menissier F, Massabanda J, Fries R, Hanset R, Georges MA. A deletion in the bovine myostatin gene causes the double-muscled phenotype in cattle. Nature Genetics 1997; 17:71-74.

8. Casas E, Keele JW, Shackelford SD, Koohmaraie M, Sonstegard TS, Smith TP, Kappes SM, Stone RT. Association of the muscle hypertrophy locus with carcass traits in beef cattle. Journal of Animal Science 1998; 76:468-473.

9. Yang J, Ratovitski T, Brady JP, Solomon MB, Wells KD, Wall RJ. Expression of myostatin pro domain results in muscular transgenic mice. Mol Reprod Dev. 2001; 60:351-361.

10. Andersson L, Georges M. Domestic-animal genomics: deciphering the genetics of complex traits. Nat Rev Genet. 2004; 5:202-212.

11. [Internet] Wong E. Poultry Research in the Post-Genome Era, Information Systems for Biotechnology News Report. February 2004. http://www.isb.vt.edu/news/2004/feb04.pdf

12. Roberts RM. The place of farm animal species in the new genomics world of reproductive biology. Biology of Reproduction 2001; 64:409-417.

13. Schmidt CW. Metabolomics: what's happening downstream of DNA. Environmental Health Perspectives 2004; 112:A410-A415.

14. Venter JC, Remington K, Heidelberg JF, Halpern AL, Rusch D, Eisen JA, et al. Environmental genome shotgun sequencing of the Sargasso Sea. Science 2004; 304:66-74.

15. Alexander DJ. Should we change the definition of avian influenza for eradication purposes? Avian Diseases. 2003;
47:976-981.

16. [Internet] US-EC Task Force on Biotechnology. Livestock Genomics Symposium Report July 2006. http://ec.europa.eu/research/biotechnology/ec-us/docs/ec-u s_workshop_animal_genomics_july_2006_en.pdf

17. [Internet] USDA-ARS. National Program 101, Food Animal Production Action Plan 2007-2012. http://www.ars.usda.gov/SP2UserFiles/Program/101/2007-2 012ActionPlan/101ActionPlan2007-2012FINAL.pdf 